\title{
Accelerated acquisition of carotid MR angiography using 3D gradient-echo imaging with two-point Dixon
}

\author{
Ryusuke Irie $^{1}$ (D) Shiori Amemiya ${ }^{1} \cdot$ Tsuyoshi Ueyama $^{2} \cdot$ Yuichi Suzuki $^{2} \cdot$ Kouhei Kamiya $^{1} \cdot$ Hidemasa Takao $^{1}$. \\ Harushi Mori $^{3}$. Osamu Abe ${ }^{1}$
}

Received: 14 February 2020 / Accepted: 28 April 2020 / Published online: 18 May 2020

(C) The Author(s) 2020

\begin{abstract}
This pilot study tests the feasibility of rapid carotid MR angiography using the liver acquisition with volume acceleration-flex technique (LAVA MRA). Seven healthy volunteers and 21 consecutive patients suspected of carotid stenosis underwent LAVA and conventional time-of-flight (cTOF) MRAs. Artery-to-fat and artery-to-muscle signal intensity ratios were manually measured. LAVA MRA exhibited a significantly larger artery-to-fat signal intensity ratio compared with cTOF MRA in all slices $(P<0.001)$ and exhibited a larger $(P<0.001)$ or equivalent $(P=1.0)$ artery-to-muscle signal intensity ratio in the extracranial carotid arteries. The image quality of the cervical carotid bifurcation and the signal change on each MRA were visually assessed and compared among the MRAs. There was no significant difference between the two MRAs in visual assessment. LAVA MRA can provide visualization similar to cTOF MRA in the evaluation of the cervical carotid bifurcation while reducing scan time by one-fifth.
\end{abstract}

Keywords MR angiography $\cdot$ Two-point Dixon $\cdot$ Scan time

\section{Introduction}

Three-dimensional (3D) time-of-flight MR angiography (TOF MRA) is a widely used non-contrast-enhanced (CE) procedure for assessing the carotid artery [1]. However, TOF MRA generally takes a few minutes to acquire and is consequently more susceptible to motion artifacts due to swallowing or gross body movement [2-4]. When imaging patients with acute symptoms or patients who have difficulty maintaining posture, image quality can be improved by reducing scan time.

Electronic supplementary material The online version of this article (https://doi.org/10.1007/s00234-020-02452-6) contains supplementary material, which is available to authorized users.

Ryusuke Irie

ririe-tky@umin.ac.jp

1 Department of Radiology, Graduate School of Medicine, The University of Tokyo, 7-3-1 Hongo, Bunkyo-ku, Tokyo 113-8655, Japan

2 Department of Radiology, The University of Tokyo Hospital, Tokyo, Japan

3 Department of Radiology, School of Medicine, Jichi Medical University, Tochigi, Japan
In this study, to reduce the scan time of carotid inflowdependent MRA, we applied the liver acquisition with volume acceleration-flex (LAVA-Flex) technique. LAVA-Flex is a 3D fast spoiled gradient-echo T1-weighted imaging technique that uses the two-point Dixon method [5]. In the LAVA-Flex technique, because the repetition time (TR) is set as short as one-sixth of the TR of the conventional TOF MRA (cTOF MRA), the scan time can be remarkably shortened.

The purposes of this study were to investigate optimal imaging conditions for clinical use of carotid MRA using LAVA-Flex (LAVA MRA) and to investigate whether LAVA MRA yields diagnostic images comparable with those of cTOF MRA in patients suspected of cervical carotid stenosis.

\section{Materials and methods}

This prospective study was approved by the Institutional Review Board (2390-(9)). Seven healthy volunteers (M:F = $6: 1$, age $30.0 \pm 4.7$ ) and 21 consecutive patients suspected of cervical carotid stenosis $(\mathrm{M}: \mathrm{F}=14: 7$, age $73.0 \pm 7.6)$ were included in this study. Written informed consent was obtained from all participants. 
All subjects underwent both cTOF and LAVA MRAs for the cervical carotid artery. For cases with cervical carotid stenosis, a 3D fat-saturated $\mathrm{T} 1$ Cube sequence was additionally scanned. MRI scans were conducted using a 3.0-T clinical scanner (Signa Premier Ver27; GE Healthcare, Milwaukee, WI, USA) with a 21-ch head-neck coil. The center of the scanning area in the cranio-caudal direction was set at $1 \mathrm{~cm}$ above the carotid bifurcation. The imaging parameters for LAVA MRA were as follows: TR $=4.9 \mathrm{~ms}$; $\mathrm{TE}=1.1 \mathrm{~ms}$ (out-of-phase) and $2.2 \mathrm{~ms}$ (in-phase); $\mathrm{FA}=4^{\circ}, 7^{\circ}$, and $10^{\circ}$; bandwidth $=1302.1 \mathrm{~Hz} /$ pixel; field of view $=220 \mathrm{~mm} \times 220$ $\mathrm{mm}$; matrix size $=256 \times 224$; parallel imaging acceleration factor $=2$; slice orientation $=$ transaxial plane; slice thickness $=2 \mathrm{~mm} \times 50$ slices (reconstruction $2 \mathrm{~mm}$ with $1 \mathrm{~mm}$ overlap); number of imaging slabs $=1$; number of excitations $=1$; scan time $=29 \mathrm{~s}$. The spatial parameters, including the positioning and acceleration factor, of cTOF MRA were set the same as those for LAVA MRA. cTOF MRA was acquired using ramped excitation pulses with flow compensation, and without fat suppression. Other factors for cTOF MRA were as follows: $\mathrm{TR}=30.0 \mathrm{~ms} ; \mathrm{TE}=3.4 \mathrm{~ms} ; \mathrm{FA}=20^{\circ}$; bandwidth $=244.1 \mathrm{~Hz} /$ pixel; scan time $=152 \mathrm{~s}$. Venous inflow saturation was applied for both LAVA and cTOF MRAs. The imaging parameters for 3D fat-saturated T1 Cube were as follows: TR $=602 \mathrm{~ms} ; \mathrm{TE}=11.9 \mathrm{~ms}$; initial $/$ minimum $\mathrm{FA}=120^{\circ} / 25^{\circ}$; bandwidth $=488.3 \mathrm{~Hz} /$ pixel; field of view $=200 \mathrm{~mm} \times 200$ $\mathrm{mm}$; matrix size $=256 \times 256($ reconstruction $512 \times 512)$; parallel imaging acceleration factors $=2.0$ (phase), 1.5 (slice); compressed sensing factor $=1.1$; slice orientation $=$ coronal plane; slice thickness $=1 \mathrm{~mm} \times 180$ slices (reconstruction $0.5 \mathrm{~mm}$ ); number of excitations $=1$; scan time $=$ $223 \mathrm{~s}$.

In order to determine the optimal flip angle (FA) for the LAVA-Flex method, imaging was performed by changing the FA to $4^{\circ}, 7^{\circ}, 10^{\circ}$, and $13^{\circ}$ in healthy volunteers.

One patient had left cervical internal carotid artery (ICA) occlusion, so region-of-interest (ROI) measurements were made on seven volunteers and 20 patients. The ROIs were placed in six slices of MRA source images. First, the slice of the carotid bifurcation was set as an index, and the slices 1 and $2 \mathrm{~cm}$ inferior and 1,2 , and $4 \mathrm{~cm}$ superior to the index slice were chosen for measurement. Six images were designated as slice 1 to slice 6 , from bottom to top. For each slice of the cTOF MRA, ROIs were manually set to cover the maximum area within the bilateral carotid arteries while avoiding the plaque (approximately $5 \mathrm{~mm}$ in diameter). The similar or larger size ROIs were placed within the bilateral sternocleidomastoid muscles (or masseter muscles) and subcutaneous fat in the posterior neck to measure the average signal. To minimize arbitrariness, all ROIs were copied to the same position in the same level of slices for other MRAs. One radiologist (experience of 7 years) and two MR technological specialists (experience of 14 years and 15 years) created ROIs, and another radiologist (experience of 14 years) checked them (Supplement 1). For each of the six slices, the signal intensity of each structure was measured and artery-tofat $\left(\mathrm{SI}_{\text {artery/fat }}\right)$ and artery-to-muscle $\left(\mathrm{SI}_{\text {artery/muscle }}\right)$ signal intensity ratios were calculated. The signal ratios measured on the left and right were averaged, and a comparison between the MRA sequences was performed for each slice.

For visual assessment, two board-certified radiologists (experience of 14 and 7 years) independently evaluated each MRA image and the final score of the visual assessment was reached by consensus. The image quality based on the shape of cervical carotid bifurcation was assessed visually with the maximum intensity projection (MIP) images ((1) nondiagnostic: the presence or absence of stenosis cannot be evaluated in some part of the bifurcation; (2) less diagnostic: partly difficult to evaluate; (3) diagnostic: easy to evaluate). The signal drop due to vortex flow at the cervical carotid bifurcation was assessed visually with the axial source images ((1) signal drop $>50 \%$ of the cross-sectional area; (2) signal drop $<50 \%$; (3) signal drop $<25 \%$; (4) no signal drop). The signal drop due to vortex flow was evaluated separately for the left and right, and for one patient with a left cervical ICA occlusion, only the right side was evaluated. Eighteen cervical carotid stenosis lesions were identified in 11 out of 21 patients. In patients with cervical carotid stenosis, the stenosis rate was measured using axial images of LAVA MRA with FA $10^{\circ}$ and cTOF MRA according to the European Carotid Surgery Trial method [6]. We measured the diameter of patent lumen and outer diameter of ICA where stenosis was the most severe on axial images. We defined the stenosis rate over $25 \%$ was positive.

Statistical analysis was performed using one-way repeatedmeasures analysis of variance (ANOVA) with the Bonferroni method for post hoc comparisons in the ROI study, the Friedman test with the Nemenyi test for post hoc comparisons in the visual assessment, and paired $t$ test in the evaluation of the cervical carotid stenosis rate. The inter-rater agreement was statistically determined by using Cohen's weighted kappa analysis. All analyses were performed using SPSS 22 software (SPSS Inc., Chicago, IL) and JMP pro 14.1 (SAS Institute, Cary, NC, USA).

\section{Results}

\section{Pilot study in normal volunteers}

LAVA MRA was imaged with FA set at $4^{\circ}, 7^{\circ}, 10^{\circ}$, and $13^{\circ}$ (Supplement 2). At a FA of $4^{\circ}$, the blood flow signal was weak and not suitable for evaluation. As the FA increased, the intravascular signal increased. However, at a FA of $13^{\circ}$, the signal drop at the bifurcation due to the vortex flow was noticeable, and the distal part of the vertebral artery was 
Table 1 Visual evaluation scores of the young healthy volunteers

\begin{tabular}{lllll}
\hline & FA 4 & FA 7 & FA 10 & cTOF \\
\hline Image quality of MIP images & $1.00 \pm 0.00$ & $3.00 \pm 0.00$ & $3.00 \pm 0.00$ & $3.00 \pm 0.00$ \\
Inhomogeneity due to the vortex flow & $3.86 \pm 0.36$ & $2.29 \pm 1.07$ & $1.86 \pm 0.53$ & $1.86 \pm 0.53$ \\
\hline
\end{tabular}

Data are mean \pm standard deviation. $F A 4^{\circ}$, flip angle $4^{\circ}$ (LAVA); $F A 7^{\circ}$, flip angle $7^{\circ}$ (LAVA); $F A 10^{\circ}$, flip angle $10^{\circ}$ (LAVA); cTOF, conventional time-of-flight; $M I P$, maximum intensity projection poorly rendered in the MIP image. Therefore, we set the FA to $7^{\circ}$ and $10^{\circ}$ for patient data acquisition (Table 1 ).

\section{ROI study and visual assessment}

ROI study of the volunteers and patients are shown in Fig. 1 and the visual evaluation scores are shown in Table 2. Representative MIP images of each sequence in the patient are shown in Fig. 2.

Repeated-measures ANOVA of $\mathrm{SI}_{\text {artery/fat }}$ and $\mathrm{SI}_{\text {artery/muscle }}$ of the volunteers $\left(F_{3,18}>5.06, P<0.01 ; F_{3,18}>53.6, P<\right.$ 0.001 , respectively) and that of the patients $\left(F_{2,38}>44.2, P<\right.$ $0.001 ; F_{2,38}>65.2, P<0.001$, respectively) found significant differences among the imaging sequences for all slices. In the volunteers, LAVA MRA with FA $10^{\circ}$ exhibited a significantly larger (slices 3-6, Bonferroni corrected $P<0.05$ ) or equivalent (slices 1 and 2) $\mathrm{SI}_{\text {artery/fat }}$ compared with cTOF MRA. LAVA MRA with FA $7^{\circ}$ also exhibited a significantly larger
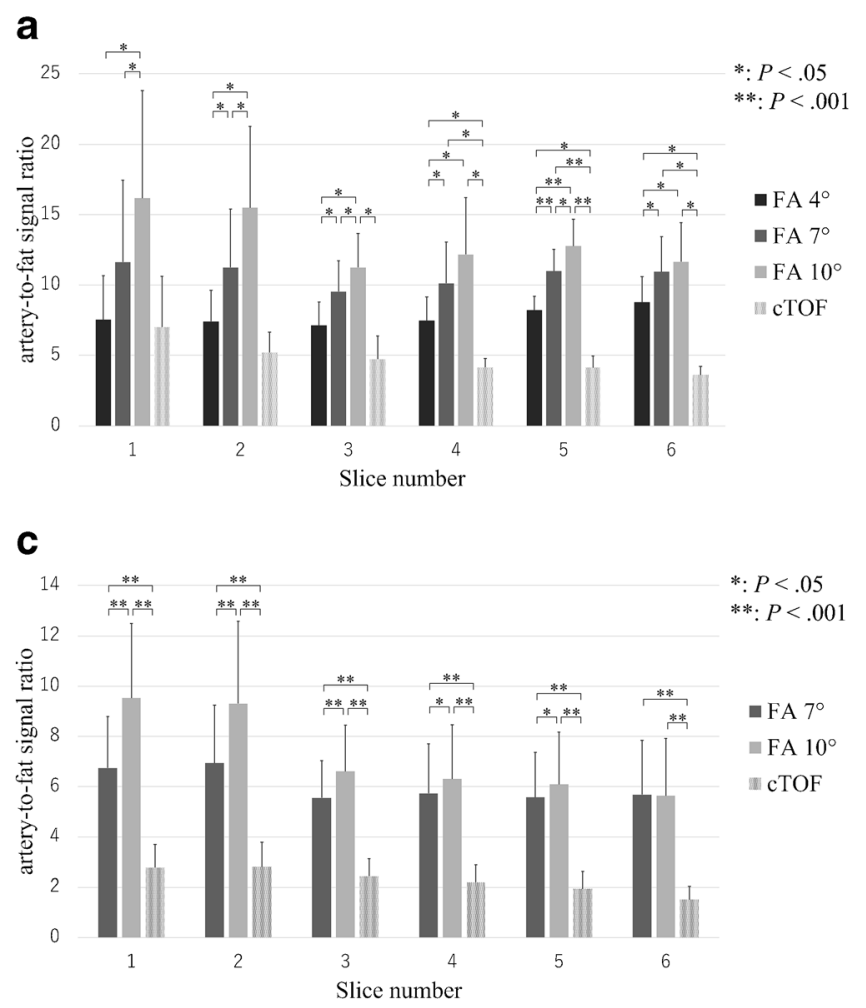

Fig. 1 Artery-to-fat and artery-to-muscle signal intensity ratios of the volunteers $(\mathbf{a}, \mathbf{b})$ and of the patients $(\mathbf{c}, \mathbf{d})$. Mean signal ratio and standard deviation in six slices of the four sequences (LAVA MRA with FA $4^{\circ}$, (slices $4-6, P<0.05$ ) or equivalent (slices $1-3$ ) signal ratio compared with cTOF MRA. LAVA MRA with FA $10^{\circ}$ exhibited a significantly larger (slices 1 and $2, P<0.001$ ) or equivalent (slices 3 and 4) $\mathrm{SI}_{\text {artery/muscle }}$ in the common carotid and proximal internal carotid artery compared with cTOF MRA, respectively. LAVA MRA with FA $7^{\circ}$ also exhibited an equivalent signal ratio in the common carotid artery compared with cTOF MRA (slices 1 and 2). By contrast, the signal ratio was significantly decreased in the distal part of the ICA (slices $3-6$ for $\mathrm{FA} 7^{\circ}$ and slices 5 and 6 for FA $10^{\circ}, P<0.05$ ). LAVA MRA with FA $4^{\circ}$ exhibited a significantly lower signal ratio compared with $\mathrm{CTOF}$ MRA $(P<0.001)$ in all slices. In the patients, LAVA MRA with FA $7^{\circ}$ and $10^{\circ}$ exhibited a significantly larger $\mathrm{SI}_{\text {artery/fat }}$ compared with cTOF MRA in all slices $(P<0.001)$. LAVA MRA with FA $10^{\circ}$ exhibited a significantly larger (slices 1 and $2, P<0.001$ ) or equivalent (slice 3 ) $\mathrm{SI}_{\text {artery/muscle }}$ in the common carotid artery and the cervical carotid bifurcation compared with cTOF MRA.
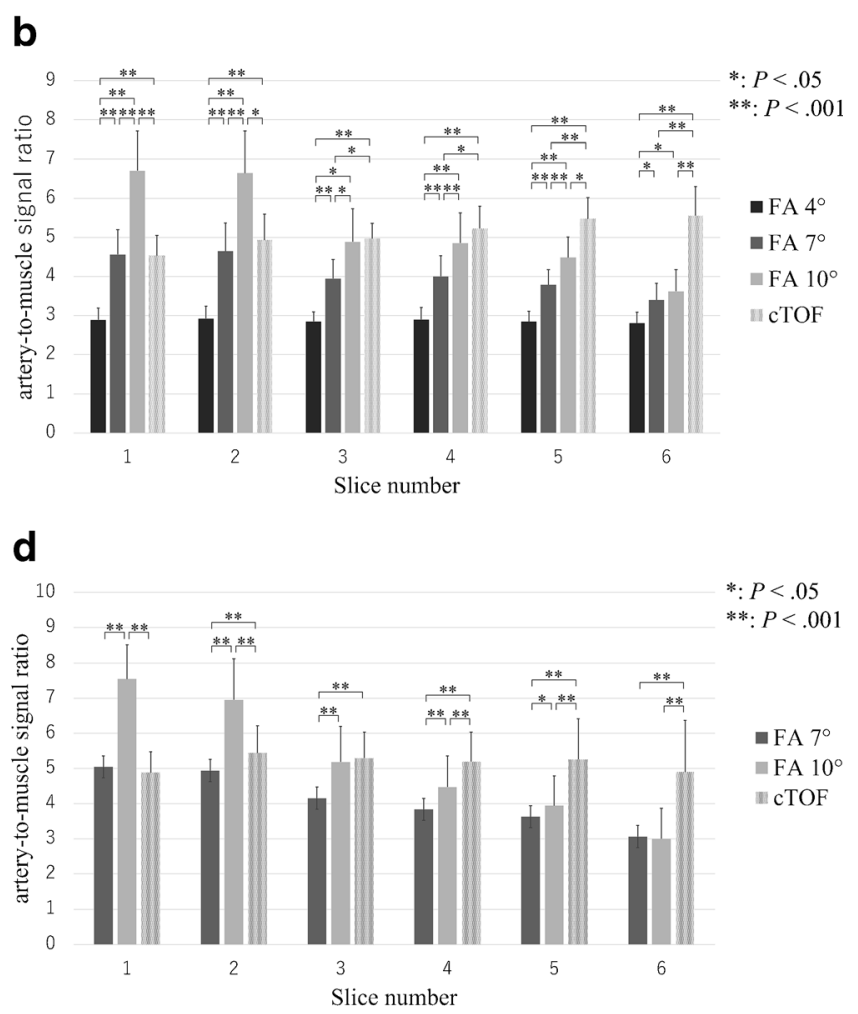

FA $7^{\circ}$, and FA $10^{\circ}$ and cTOF MRA) for volunteer data and three sequences (LAVA MRA with FA $7^{\circ}$ and FA $10^{\circ}$ and cTOF MRA) for patient data are shown as a bar graph. $* P<0.05 ; * * P<0.001$ 
Table 2 Visual evaluation scores of the patients

\begin{tabular}{llll}
\hline & FA 7 & FA 10 & cTOF \\
\hline Image quality of MIP images & $2.75 \pm 0.44$ & $2.81 \pm 0.40$ & $2.86 \pm 0.48$ \\
Inhomogeneity due to the vortex flow & $2.56 \pm 0.95$ & $2.34 \pm 0.88$ & $2.42 \pm 0.89$ \\
\hline
\end{tabular}

Data are mean \pm standard deviation. $F A 7^{\circ}$, flip angle $7^{\circ}$ (LAVA); FA $10^{\circ}$, flip angle $10^{\circ}$ (LAVA); cTOF, conventional time-of-flight; $M I P$, maximum intensity projection
LAVA MRA with FA $7^{\circ}$ also exhibited an equivalent $\mathrm{SI}_{\text {artery/ }}$ muscle in the common carotid artery compared with cTOF MRA (slice 1). By contrast, a significantly decreased $\mathrm{SI}_{\text {artery/ }}$ muscle was seen in the more distal part (slices 2-6 for FA $7^{\circ}$ and slices 4-6 for FA $\left.10^{\circ}, P<0.001\right)$.

In the visual assessment of the volunteers, there were significant differences among the four sequences about the scores of image quality based on the shape of carotid bifurcation (Friedman $\chi^{2}=21.0, P<0.001$ ) and about the inhomogeneity scores due to the vortex flow $\left(\chi^{2}=35.4, P<0.001\right)$. The signal was significantly more homogeneous for FA $4^{\circ}(P<$ $0.01)$ due to a low overall arterial signal, whereas it was not significantly different for FA $7^{\circ}, \mathrm{FA} 10^{\circ}$, and cTOF $(P>0.81$, respectively). In the patients, there was no significant difference among the three sequences about the scores of image quality based on the shape of carotid bifurcation $\left(\chi^{2}=2.33\right.$, $P=0.31$; Nemenyi multiple comparisons, $P>0.77)$ and there was no significant difference among them about the scores of inhomogeneity due to the vortex flow $\left(\chi^{2}=3.71, P=0.15\right.$; Nemenyi multiple comparisons, $P>0.79$ ). Weighted kappa coefficient was 0.73 in the image quality evaluation and 0.67 in the signal drop evaluation.

Comparison of cervical carotid stenosis rates showed no significant difference between LAVA MRA with FA $10^{\circ}$ and cTOF MRA $(0.47 \pm 0.17$ vs. $0.46 \pm 0.21, P=0.64)$. When the stenosis rate was classified as mild $(<50 \%)$, moderate $(50-69 \%)$, or severe $(\geq 70 \%)$, the results of the two sequences were completely consistent. In one case with severe stenosis, LAVA MRA was able to clearly identify the signal of the thin patent lumen (Supplement 3). When a signal defect caused by the jet-flow was recognized in the stenosis part, it was determined that the part was patent and the stenosis rate was measured (Supplement 4).

\section{Discussion}

When the LAVA-Flex method in which the carotid MRA scanning is completed in only $29 \mathrm{~s}$ is adopted, the effect of time reduction is very large. Several reports have used parallel imaging or compressed sensing to reduce the time needed to complete carotid MRA [7, 8]. LAVA MRA can be acquired in a very short time compared with these methods.

Compared with cTOF MRA, arterial signal loss at the distal part of the ICA was greater with LAVA MRA, as expected. CTOF MRA applies a ramped excitation pulse to modulate the FA in order to prevent signal degradation at the distal end of the ICA $[9,10]$. LAVA-Flex used in this study is a commercial sequence for the upper abdomen, so the improvement of image quality may be expected by creating a pulse sequence optimized for blood vessels, such as by introducing ramped excitation or by increasing the TR as long as the scan time can be kept short.
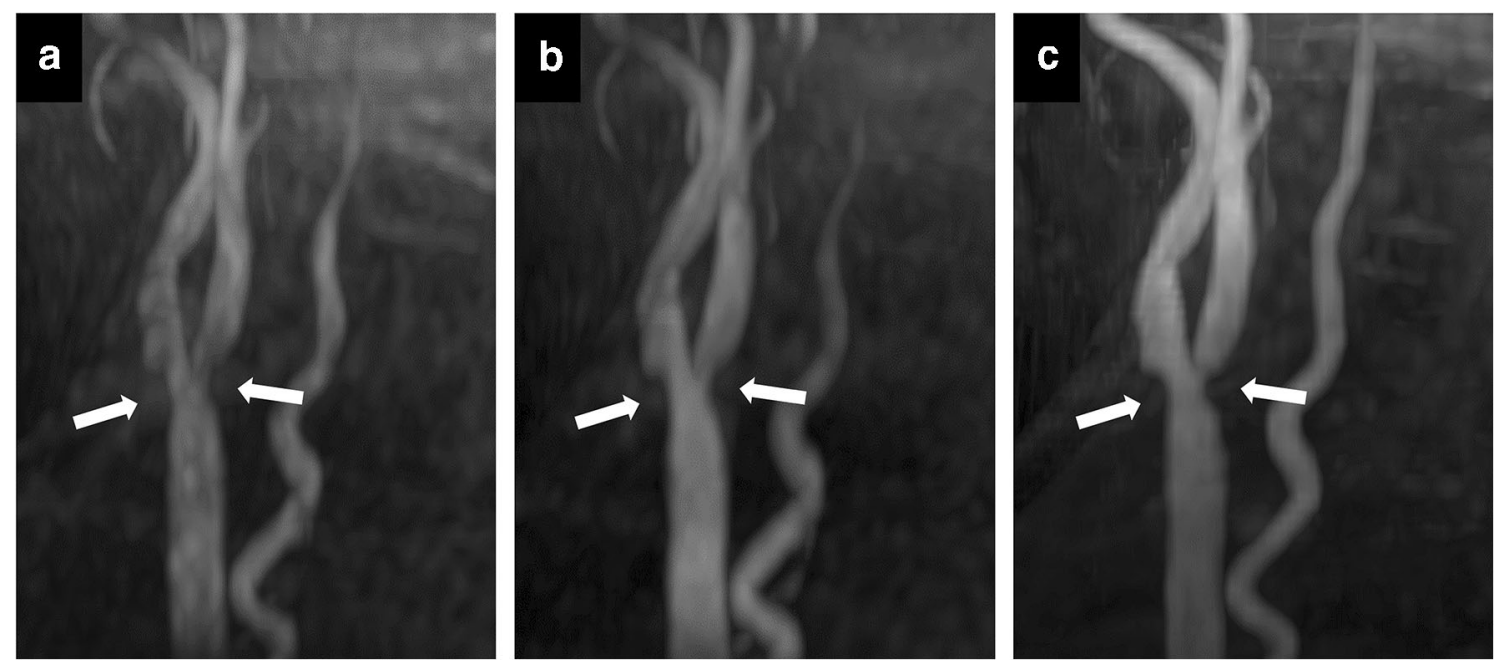

Fig. 2 MIP images of a patient with cervical carotid stenosis. LAVA MRA with FA $7^{\circ}$ (a) or FA $10^{\circ}$ (b) can identify the shape of cervical carotid stenosis as effectively as cTOF MRA (c) 
In this study, we set the cTOF MRA used in routine clinical practices as a reference standard. Non-CE MRA can overestimate the stenosis, mainly due to the vortex flow or the jetflow at the site of severe stenosis. The lack of CE examinations can be a limitation of this study, but overestimation can also occur with CE MRA, which is sometimes worse than with non-CE MRA [11].

In conclusion, LAVA MRA can provide information similar to cTOF MRA for assessing the cervical carotid bifurcation while reducing scan time by one-fifth.

Funding information This study was funded by Grant-in-Aid for Scientific Research from Japanese Society of Neuroradiology.

\section{Compliance with ethical standards}

Conflict of interest The authors declare that they have no conflict of interest.

Ethical approval All procedures performed in studies involving human participants were in accordance with the ethical standards of the institutional research committee and with the 1964 Helsinki declaration and its later amendments or comparable ethical standards.

Informed consent Informed consent was obtained from all individual participants included in the study.

Open Access This article is licensed under a Creative Commons Attribution 4.0 International License, which permits use, sharing, adaptation, distribution and reproduction in any medium or format, as long as you give appropriate credit to the original author(s) and the source, provide a link to the Creative Commons licence, and indicate if changes were made. The images or other third party material in this article are included in the article's Creative Commons licence, unless indicated otherwise in a credit line to the material. If material is not included in the article's Creative Commons licence and your intended use is not permitted by statutory regulation or exceeds the permitted use, you will need to obtain permission directly from the copyright holder. To view a copy of this licence, visit http://creativecommons.org/licenses/by/4.0/.

\section{References}

1. Debrey SM, Yu H, Lynch JK, Lovblad KO, Wright VL, Janket SJ et al (2008) Diagnostic accuracy of magnetic resonance angiography for internal carotid artery disease: a systematic review and meta-analysis. Stroke 39(8):2237-2248. https://doi.org/10. 1161/strokeaha.107.509877

2. Heiserman JE, Drayer BP, Keller PJ, Fram EK (1992) Intracranial vascular stenosis and occlusion: evaluation with three-dimensional time-of-flight MR angiography. Radiology 185(3):667-673. https://doi.org/10.1148/radiology.185.3.1438743

3. Maas K, Barkovich AJ, Dong L, Edwards MS, Piecuch RE, Charlton V (1994) Selected indications for and applications of magnetic resonance angiography in children. Pediatr Neurosurg 20(2): 113-125. https://doi.org/10.1159/000120774

4. Grist TM, Thornton FJ (2005) Magnetic resonance angiography in children: technique, indications, and imaging findings. Pediatr Radiol 35(1):26-39. https://doi.org/10.1007/s00247-004-1350-1

5. Ma J (2004) Breath-hold water and fat imaging using a dual-echo two-point Dixon technique with an efficient and robust phasecorrection algorithm. Magn Reson Med 52(2):415-419. https:// doi.org/10.1002/mrm.20146

6. European Carotid Surgery Trialists' Collaborative Group (1991) MRC European Carotid Surgery Trial: interim results for symptomatic patients with severe (70-99\%) or with mild (0-29\%) carotid stenosis. Lancet 337(8752):1235-1243

7. Chen S, Ning J, Zhao X, Wang J, Zhou Z, Yuan C, Chen H (2017) Fast simultaneous noncontrast angiography and intraplaque hemorrhage (fSNAP) sequence for carotid artery imaging. Magn Reson Med 77(2):753-758. https://doi.org/10.1002/mrm.26111

8. Li B, Li H, Dong L, Huang G (2017) Fast carotid artery MR angiography with compressed sensing based three-dimensional time-offlight sequence. Magn Reson Imaging 43:129-135. https://doi.org/ 10.1016/j.mri.2017.07.017

9. Atkinson D, Brant-Zawadzki M, Gillan G, Purdy D, Laub G (1994) Improved MR angiography: magnetization transfer suppression with variable flip angle excitation and increased resolution. Radiology 190(3):890-894. https://doi.org/10.1148/radiology.190. 3.8115646

10. Ding X, Tkach JA, Ruggieri PR, Masaryk TJ (1994) Sequential three-dimensional time-of-flight MR angiography of the carotid arteries: value of variable excitation and postprocessing in reducing venetian blind artifact. AJR Am J Roentgenol 163(3):683-688. https://doi.org/10.2214/ajr.163.3.8079868

11. Townsend TC, Saloner D, Pan XM, Rapp JH (2003) Contrast material-enhanced MRA overestimates severity of carotid stenosis, compared with 3D time-of-flight MRA. J Vasc Surg 38(1):36-40. https://doi.org/10.1016/s0741-5214(03)00332-x

Publisher's note Springer Nature remains neutral with regard to jurisdictional claims in published maps and institutional affiliations. 